\title{
Synthèse ultrarapide de nanoparticules par claquage dans des liquides diélectriques
}

Thierry Belmonte (thierry.belmonte@univ-lorraine.fr)

Institut Jean Lamour, Département CP2S, UMR 7198 CNRS-Université de Lorraine,

Parc de Saurupt, CS 50840, 54011 Nancy Cedex

Les récents progrès sur la

compréhension des décharges

produites dans les liquides

diélectriques entre deux

électrodes dont on choisit

la composition, ouvrent

des perspectives nouvelles

dans la maîtrise de l'élaboration

de nanoparticules. De telles

décharges permettent de

synthétiser des nanoparticules

ayant des diamètres

caractéristiques compris

entre 2 et $10 \mathrm{~nm}$. Surtout,

elles permettent d'atteindre

des rendements de production

remarquablement élevés,

de l'ordre de la centaine

de grammes par heure.

La difficulté principale

consiste à maîtriser ce procédé

qui est basé sur un phénomène

stochastique : le claquage

d'un diélectrique, qui permet

la formation d'une décharge

électrique.
L'étude des décharges produites entre deux électrodes dans des liquides diélectriques a longtemps été l'apanage des électrotechniciens, qui cherchaient à améliorer les disjoncteurs de forte puissance. En observant que les électrodes s'érodaient lors de chaque décharge, les ingénieurs ont pensé à exploiter ce phénomène pour améliorer l'usinage des métaux. C'est ainsi que l'usinage par électroérosion est apparu et a été développé pour atteindre aujourd'hui des records de résolution, avec une précision de l'ordre $\mathrm{du}$ micromètre au niveau industriel. Des dispositifs basés sur des microscopes à force atomique permettent même d'obtenir aujourd'hui des nanotrous en laboratoire. La pointe de l'instrument est alors utilisée comme électrode de haute tension pour éroder la contre-électrode à très petite échelle.

Plus récemment, ce domaine a connu un regain d'intérêt en raison de l'apparition de nouvelles applications potentielles comme la synthèse de nanoparticules, l'oxydation électrolytique d'alliages métalliques ou le traitement de l'eau et des effluents. Ces développements s'accompagnent d'une redécouverte de ces décharges à haute pression, dont la physique nécessite d'être approfondie. En effet, les décharges dans les liquides créées entre deux électrodes ont un comportement fondamentalement stochastique : il est impossible de reproduire une décharge exactement à l'identique, effet qui a longtemps limité leur étude par détection synchrone. Cela résulte principalement de l'étape liée à l'injection de charges, qui sera décrite ultérieurement. Néanmoins, l'apparition de générateurs d'impulsions nanoseconde et même picoseconde fonctionnant à haute tension (de 1 à $100 \mathrm{kV}$ suivant les cas) a permis d'améliorer grandement la caractérisation de ces milieux ionisés, comme nous allons le voir.

\section{Phénoménologie des décharges}

La formation d'une décharge dans un liquide diélectrique implique un nombre important de phénomènes, de sorte qu'en démêler l'écheveau est particulièrement complexe. On distingue cinq étapes principales au cours d'un événement, chacune associée à un comportement électrique spécifique (fig. 1) :

1) La première phase consiste à mettre le liquide sous contrainte mécanique, en élevant la tension jusqu'à une valeur qui permet de réaliser la rupture du diélectrique. Typiquement, pour l'eau distillée, le champ électrique de claquage est de l'ordre de quelques MV. $\mathrm{cm}^{-1}$.

2) La phase de préclaquage correspond à une injection de charges du métal vers le liquide, qui se traduit par des oscillations de la tension électrique (encart, fig. 1). Elle peut s'accompagner, sur des temps de quelques dizaines de nanosecondes, d'un mouvement du liquide induit par un phénomène électrohydrodynamique (distinct du phénomène d'électrostriction qui se produit sur des temps plus courts, inférieurs à la nanoseconde), au cours duquel des particules ionisées lourdes vont se déplacer sous l'effet du champ électrique et déplacer avec elles le liquide alentour. Cette étape contribue, par des mécanismes différents selon la vitesse de montée en tension, à créer une zone de moindre densité, qui va permettre l'amorçage de la décharge.

3) La phase d'amorçage correspond à l'émission par le liquide comprimé 
Distribution spatiale de l'émission de lumière à $15 \mathrm{kV}$, lors d'une décharge dans l'heptane.

(a) Intensité enregistrée après le claquage, avec une caméra ultrarapide. Résolution temporelle : $1,9 \mu \mathrm{s}$.

(b) Intensité enregistrée après le claquage, avec une caméra iCCD. Résolution temporelle : 2,0 ns.
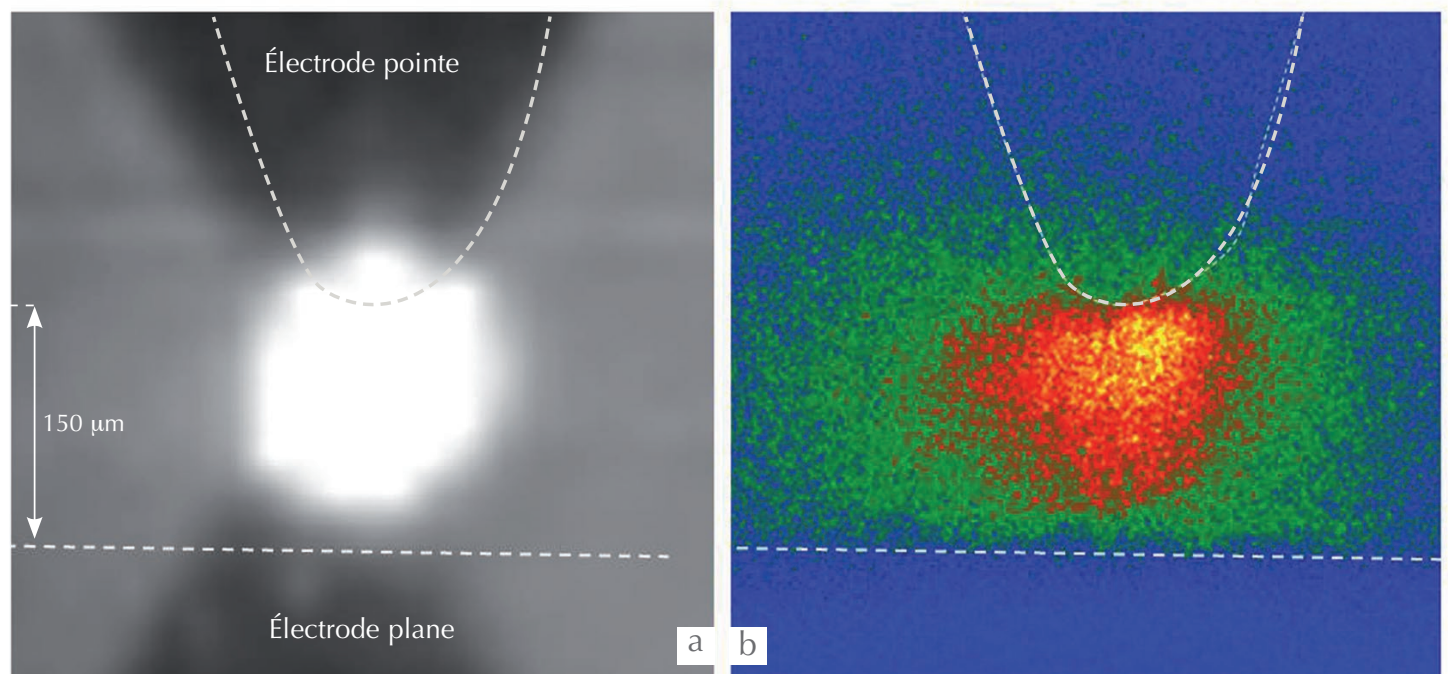

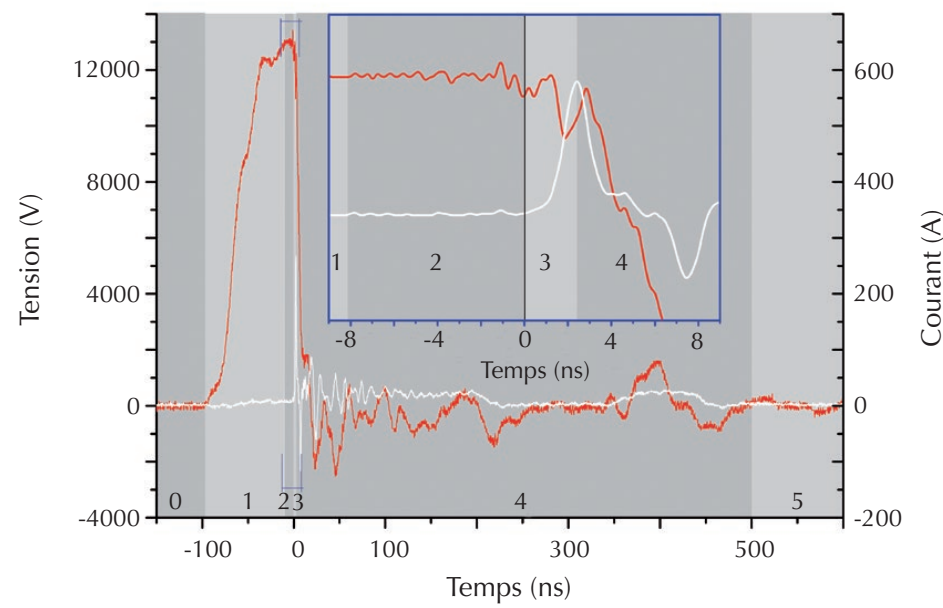

0 : Liquide au repos

1 : Montée en tension

2 : Préclaquage

3 : Streamer

4 : Décharge spark

5 : Postdécharge



$\mathrm{t}=2 \mathrm{~ns}$
4 : Spark

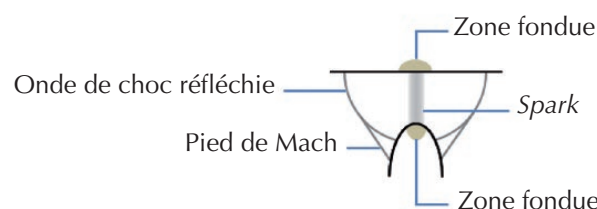

3 : Streamer

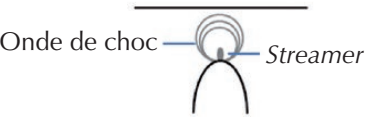

\section{5 : Bulle de cavitation}

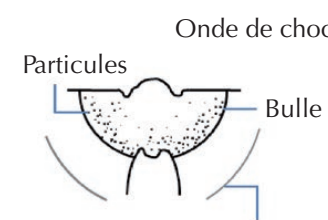

Onde de choc réfléchie

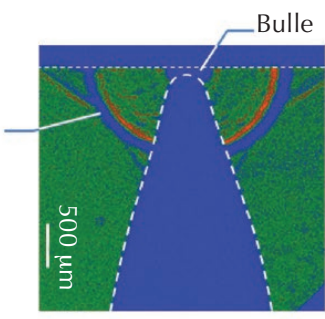

$\mathrm{t}=467 \mathrm{~ns}$
2. Schématisation des différentes étapes d'une décharge en condition pointe-plan. Le temps d'origine est défini lorsque l'intensité du courant mesuré entre les électrodes se met à croître fortement. La phase de préclaquage est associée à une injection de charges. Le développement du streamer a lieu après l'émission d'une onde de choc, avec des vitesses pouvant atteindre plusieurs $\mathrm{km} \cdot \mathrm{s}^{-1}$. Expérimentalement (image en haut à droite), on observe au moyen d'une caméra intensifiée l'amorçage du streamer mais pas l'onde de choc, en raison de l'émission lumineuse intense du plasma qui masque le rayonnement de la lampe flash servant à l'observer par ombroscopie (du rouge au bleu : intensité d'émission optique décroissante).

Lors de la phase de postdécharge, l'absence de rayonnement issu du plasma permet la visualisation de l'onde de choc après qu'elle a été réfléchie sur l'électrode plane. Sur l'image en bas à droite, on distingue nettement la bulle qui a succédé à la décharge et commence une phase d'expansion (du rouge au vert puis au bleu, densité du milieu croissante). 


\section{Oscillations d'une bulle de cavitation}

Une bulle de cavitation oscille au cours du temps et voit son diamètre augmenter puis décroître plusieurs fois avant de se stabiliser (fig. E1). Si la pression initiale dans la bulle n'est pas trop forte, les propriétés thermodynamiques du milieu ne dépendent pas de la pression. L'évolution du rayon de la bulle est alors fournie par l'équation de Rayleigh-Plesset : $R \ddot{R}+3 \dot{R} / 2=P_{i} / \rho_{0}$, qui relie les dérivées temporelles $\dot{R}$ et $\ddot{R}$ du rayon $R$ de la bulle à la pression initiale $P_{i}$ et à la densité du liquide $\rho_{0}$. En revanche, lorsque la pression est élevée, la variation des propriétés du milieu avec la pression est marquée, et il faut recourir à une autre équation, l'équation de Gilmore, pour décrire le mouvement de la bulle.

La dynamique de I'interface gaz-liquide est affectée par différents phénomènes. Par exemple, au voisinage d'une paroi, la quantité de mouvement du liquide entre la bulle et la paroi est différente de celle au-dessus de la bulle. Il faut alors prendre en compte ce que l'on nomme l'impulsion de Kelvin pour décrire la dynamique spécifique de la bulle dans ce cas. La génération d'une nouvelle bulle, si elle a lieu suffisamment tôt, induit un champ de pression dans le liquide qui peut aussi conduire à modifier la forme de la bulle précédente, voire même à la fragmenter.
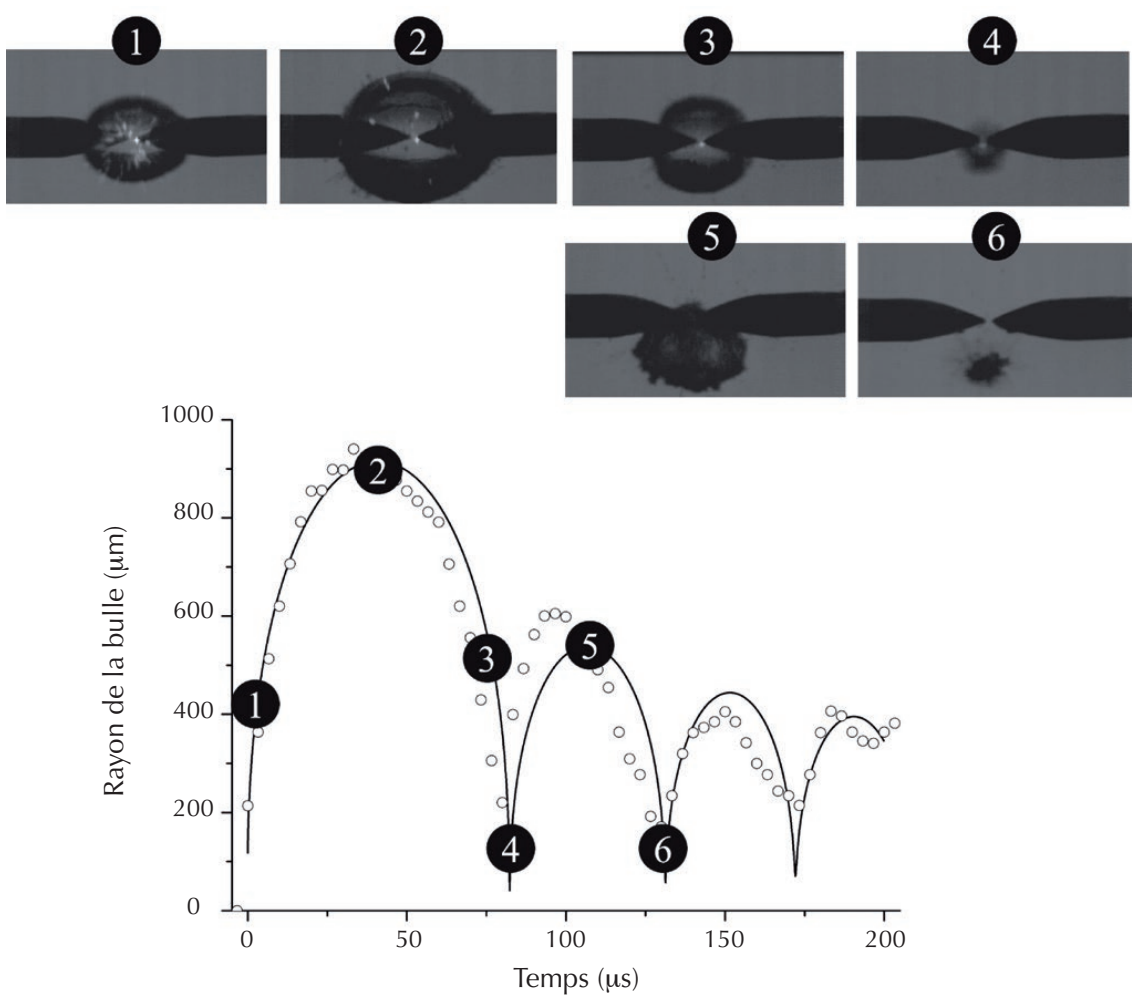

E1. Évolution temporelle du rayon d'une bulle produite à partir d'une décharge spark entre deux électrodes en forme de pointe. La bulle circonvient les électrodes et son diamètre, dans le plan de la photo, se confond avec l'axe interélectrode. II s'agit d'un cas semblable à ceux observés sur les figures 1 et 2. En trait continu, on indique le résultat du modèle de Rayleigh-Plesset. Les ronds blancs désignent les points expérimentaux mesurés dans ce cas. Ce phénomène a été observé au moyen d’une caméra rapide ayant une résolution de 500000 images par seconde. On observe une succession de phases d'expansion et de contraction. L'interface gaz-liquide, qui est le siège d'instabilités, se déforme au cours du temps. Dans les photos, les électrodes apparaissent en sombre et le liquide apparaît en clair.
\〉

d'une onde de choc et à la formation, à l'arrière de cette onde où la densité est plus faible, de ce que l'on nomme un streamer, c'est-à-dire une décharge filamentaire transitoire qui part d'une électrode et se propage vers la contreélectrode. Ce streamer - de quelques $\mu \mathrm{m}$ de diamètre - est un milieu dense, où la pression peut atteindre quelques centaines de MPa. Le courant augmente alors fortement et peut atteindre des centaines, voire des milliers d'ampères suivant la puissance du générateur utilisé. Lorsque l'espace entre les électrodes est assez grand, la propagation du streamer donne lieu à du branchage, c'est-à-dire une division du streamer père en plusieurs streamers fils, comme cela se produit communément avec la foudre. Plusieurs modes de propagation peuvent alors être observés selon la vitesse du streamer : on parle de propagation en forme de buisson, d'arbre ou de filament. Si l'espace entre les électrodes est trop grand, le streamer peut cesser de se propager à une distance appelée distance d'arrêt.

4) Lorsque le streamer atteint la contreélectrode, la tension chute fortement du fait de l'établissement d'un canal de plasma conducteur qui correspond à ce que l'on nomme une décharge spark, laquelle se distingue d'une décharge d'arc essentiellement par sa durée. On considère en effet que la décharge spark n'atteint pas l'état d'équilibre thermodynamique local, état qui caractérise les décharges d'arc. Le courant chute et se met à osciller à une fréquence de quelques MHz. Les électrodes se mettent à fondre, les températures pouvant alors atteindre quelques milliers de degrés, et émettent une vapeur métallique qui va modifier le plasma et dont la condensation va conduire à la formation de particules pour la plupart nanométriques.

5) Lorsque la décharge s'arrête, l'énergie contenue dans le volume du plasma (gaz ionisé) se relaxe en produisant une bulle de cavitation - i.e. sans élévation sensible de la température du liquide environnant - qui va se mettre à osciller selon une dynamique qui répond au premier ordre à l'équation de RayleighPlesset (voir encadré), suivant des phases de contraction et d'expansion qui pourront être le siège d'instabilités complexes de l'interface gaz-liquide. Surtout, suite à des travaux récents [4] que l'on décrira plus loin, il se trouve 
que la manière dont les nanoparticules formées passent depuis la bulle de gaz vers le liquide diélectrique est totalement différente de ce que l'on imaginait jusqu'à il y a peu.

Tous ces phénomènes sont fortement affectés par la polarité de la tension appliquée, selon qu'elle est positive ou négative, par la polarisabilité du liquide utilisé, sa pureté, etc. Récemment, de nouvelles études ont été engagées pour étudier les premiers instants du claquage dans de l'eau soumise à des impulsions picosecondes de l'ordre de $200 \mathrm{kV}$. À cette échelle, toute expansion hydrodynamique du liquide est impossible parce que la durée de l'impulsion est trop courte. Par conséquent, la densité du milieu reste proche de sa valeur initiale et la décharge est formée directement dans la phase liquide sans qu'il y ait transformation de phase [1]. Lorsque la durée de l'impulsion augmente, l'existence d'une zone de compression induite par électrostriction a pu être démontrée [2]. Cette zone de haute densité est suivie d'une autre, dite de raréfaction, qui serait alors le siège de l'amorçage du streamer dans ces conditions très particulières. Sur des échelles de temps plus longues, une couche de vapeur se forme autour de l'électrode et permet l'amorçage du streamer [3].

L'une des questions fondamentales qui se posent sitôt que l'on cherche à modéliser la formation d'un streamer est celle du comportement d'un milieu ionisé porté à très haute pression. Peu d'approches convaincantes sont disponibles à ce jour. Une difficulté majeure réside dans le fait que le milieu est optiquement épais lors des premiers instants de la décharge, du fait de la haute pression qui y règne. La lumière produite par le streamer est en grande partie piégée dans le milieu dont la densité contribue à élargir les transitions optiques, de sorte que l'observation de la lumière émise se limite à de larges continuums dont l'origine est complexe à établir. L'information fournie par l'émission de lumière est alors très pauvre. En effet, plusieurs phénomènes d'importances similaires peuvent être la source de la lumière émise, comme l'émission de corps noir (dû à l'émission de lumière produite par un corps chaud), le rayonnement de bremsstrahlung (dit aussi rayonnement de freinage, dû aux électrons libres entrant en collision avec les ions) ou le rayonnement

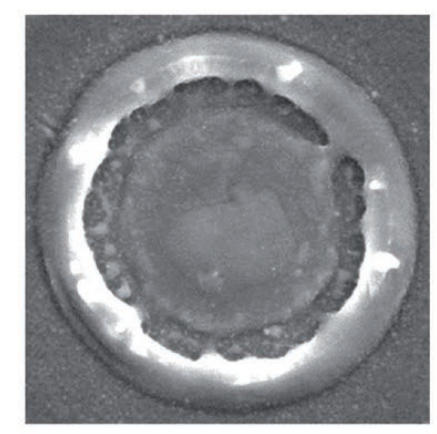

$a$

$\mathrm{b}$
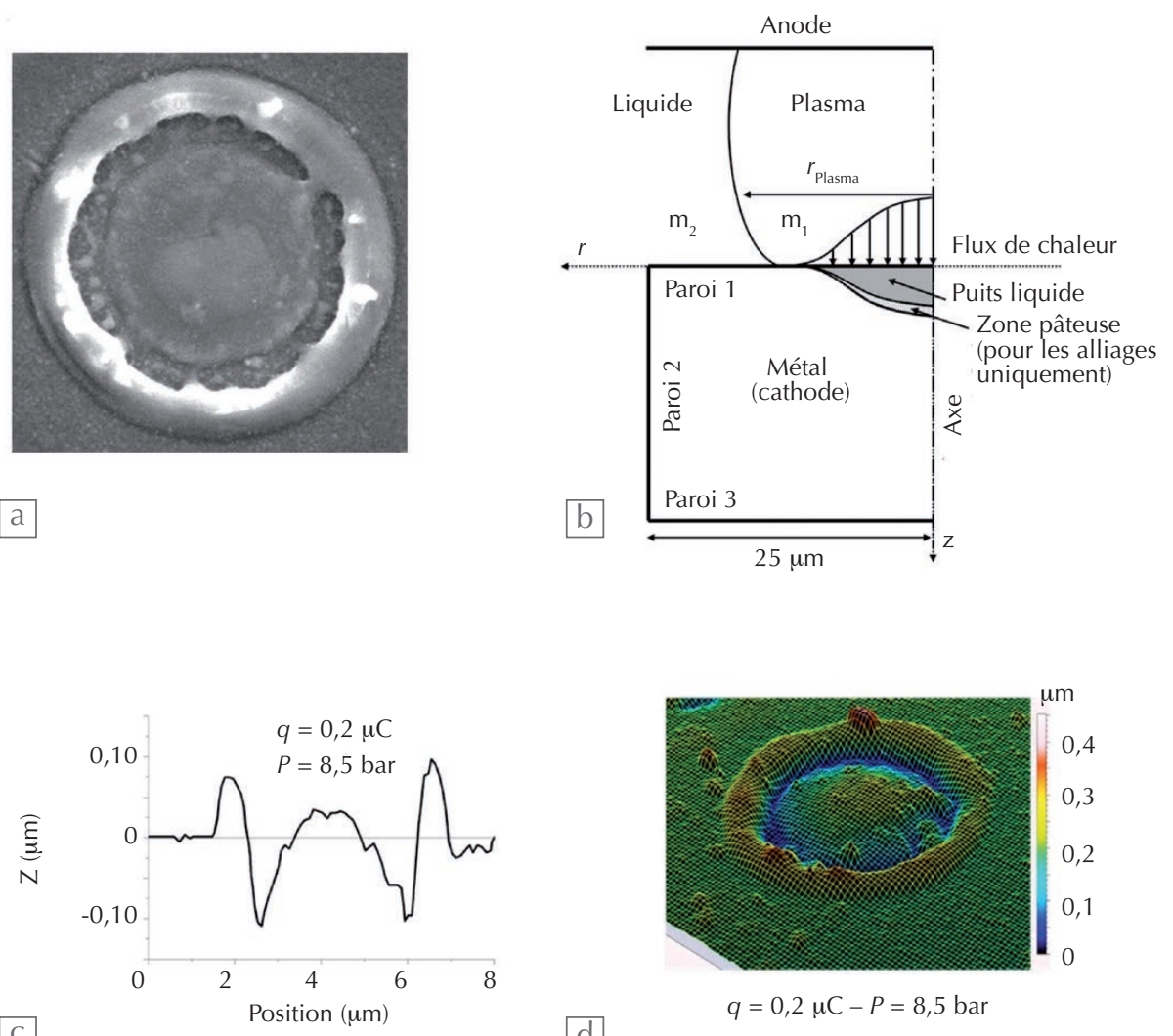

d

3. L'interaction d'une décharge spark conduit à la formation d'une zone érodée sur la surface, dont on peut apprécier en a) une vue de dessus obtenue par microscopie électronique à balayage. L'impact est généralement caractérisé par un bourrelet externe formé par effet Thomson-Marangoni. L'interaction déchargesurface peut être décrite qualitativement et de manière simplifiée selon le schéma b). $m_{1}$ et $m_{2}$ désignent respectivement le plasma, au-dessus du puits de métal liquide, et le liquide diélectrique qui est ici de l'eau distillée. La distance interélectrode est de $100 \mu \mathrm{m}$. Les électrodes sont en aluminium. Des mesures par microscopie à force atomique permettent d'accéder à des profils 2D en c) et 3D en d), afin d'estimer précisément le volume de matière déplacée ou érodée. q désigne la charge délivrée par le générateur à la décharge. P est la pression initiale estimée du plasma.

issu de la recombinaison électron-ion, notamment. Par conséquent, on ne dispose que de peu d'information sur cette étape de la décharge. De plus, elle est totalement hors équilibre et nécessite un traitement adapté qui ne peut être celui que l'on applique classiquement pour modéliser un arc, lequel nous l'avons vu est à l'équilibre. L'utilisation de l'équation de Saha notamment, qui établit le lien entre température, densité et degré d'ionisation d'un plasma à l'équilibre, est proscrite dans ce cas.

Enfin, des phénomènes spécifiques se produisent du fait des très fortes pressions appliquées. Par exemple, l'énergie d'ionisation des atomes diminue, de sorte que l'énergie requise pour former $\mathrm{H}^{+}$n'est plus de $13,6 \mathrm{eV}$, mais de $8,7 \mathrm{eV}$ à une pression de 1000 atm et une température de $10000 \mathrm{~K}$. C'est la raison pour laquelle seule la transition $\mathrm{H} \alpha$ de la série de Balmer est observable dans le cas des décharges dans l'eau, les autres transitions comme $H \beta, H \gamma$, etc. ayant lieu à partir d'états excités dont l'énergie est alors supérieure à celle de l'ion.

\section{Formation des nanoparticules}

Il n'est donc pas étonnant, à la lumière de ce qui vient d'être décrit, que la décharge formée puisse, en raison des conditions extrêmes dont elle est le siège - forte température, forte pression -, éroder les électrodes qui servent à la générer, comme le montre la figure 3. 
La synthèse de nanoparticules à partir des vapeurs produites par l'érosion des électrodes fait actuellement l'objet de nombreuses études. L'interaction spark-surface présente beaucoup de points communs avec l'interaction arc sous vide-surface, utilisée dans certains procédés industriels pour purifier les métaux (refusion à l'arc sous vide) ou pour déposer des films minces (dépôt par arc cathodique).

L'électrode soumise à un courant intense et à la radiation de la décharge s'élève en température et se met à fondre. Si l'électrode est un alliage, le puits liquide formé est séparé de la phase solide par une région dite pâteuse, dont la description est complexe. Le bain liquide est brassé par la force de Thomson-Marangoni qui résulte de la variation de tension superficielle du liquide induite par un gradient thermique. Si le courant est suffisamment intense, le champ magnétique induit peut également contribuer à brasser le liquide. Enfin, la pression du plasma peut permettre l'éjection de particules d'une taille en général submicrométrique (de quelques centaines de $\mathrm{nm}$ ). Lorsque le plasma n'est plus entretenu, c'est-à-dire lorsque le courant s'annule, la température décroît très rapidement $\left(\sim 10^{8} \mathrm{~K} \mathrm{~s}^{-1}\right)$ et le bain liquide se fige pour adopter une géométrie finale qui est du type de celle présentée en figure 3.

Ces différents phénomènes conduisent à la formation de trois familles de particules : - des nanoparticules de diamètres compris entre 2 et $10 \mathrm{~nm}$ et formées dans la décharge ;

- des particules de l'ordre de 20 à $100 \mathrm{~nm}$ synthétisées en dehors de la décharge, dans les zones plus denses à l'interface avec le liquide ;

- des particules micrométriques produites par l'éjection de métal fondu hors du puits liquide.

Des travaux récents [4] exploitant la diffusion des rayons $\mathrm{X}$ aux petits angles et réalisés au moyen d'un rayonnement synchrotron ont permis de montrer que la plupart des nanoparticules étaient piégées dans la bulle de cavitation et ne passaient pas dans le liquide lors de la contraction de la bulle, contrairement à la vision qui était admise jusqu'ici. Ce résultat est essentiel, car il explique que l'agglomération des nanoparticules est dramatiquement plus efficace que celle qui se produit dans le seul liquide.
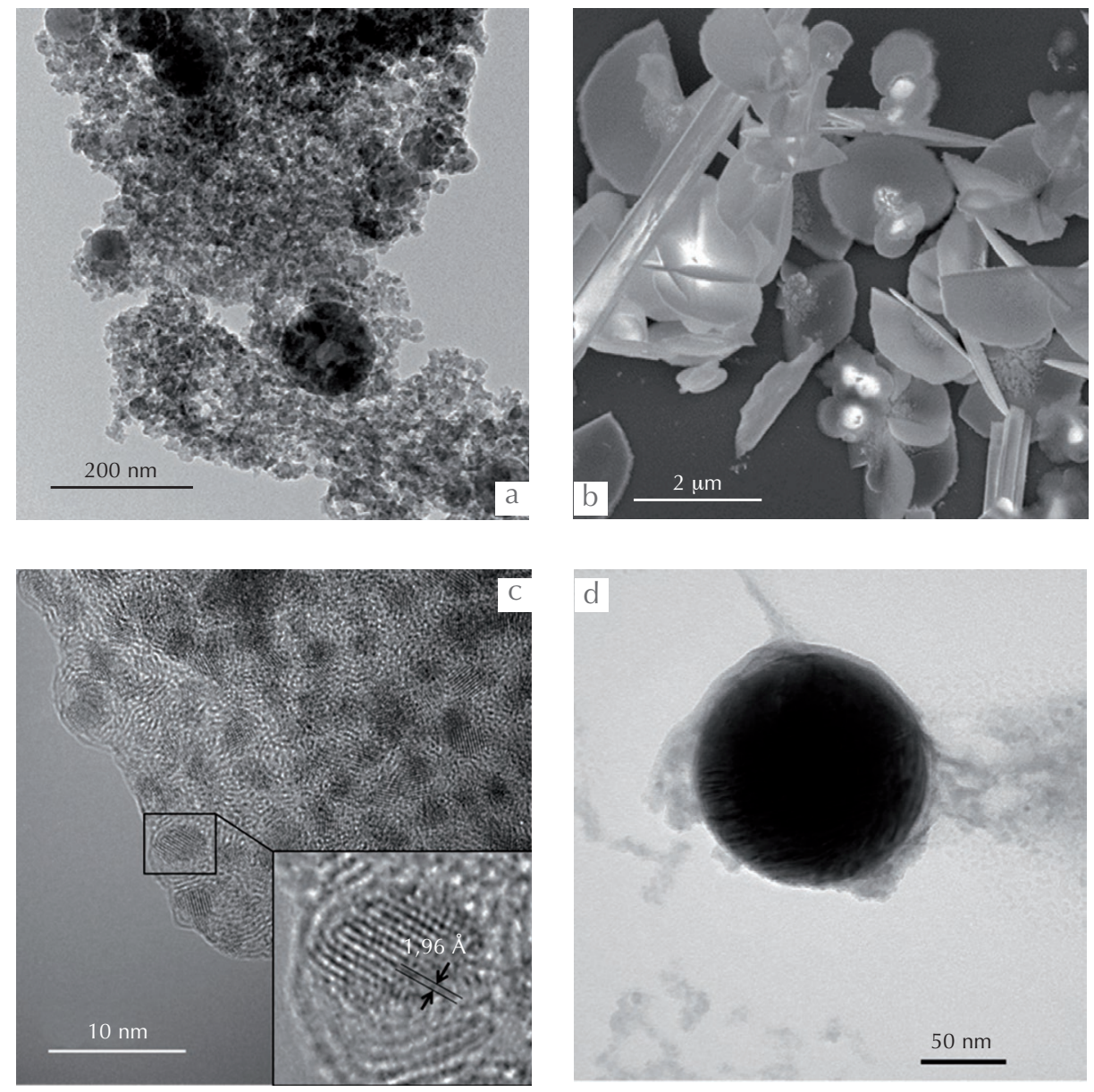

4. Exemples de nanoparticules formées par des décharges en phase liquide.

a) Nanoparticules d'alumine formées dans l'eau entre deux électrodes d'aluminium. On distingue nettement deux distributions de tailles : $100 \mathrm{~nm}$ pour les sphères gris foncé et $\sim 5 \mathrm{~nm}$ pour les petits grains autour.

b) Nanofeuillets d'oxyde de plomb formés dans l'azote liquide entre deux électrodes de plomb.

c) Nanoparticules de platine (taches sombres) dans une matrice de carbone amorphe hydrogéné (zone plus claire) formées dans l'heptane, un alcane de formule $\mathrm{C}_{7} \mathrm{H}_{16}$, entre deux électrodes de platine. Encart : zoom d'une nanoparticule montrant les plans atomiques de platine séparés de 1,96 $\AA$.

d) Nanocomposite formé d'un cœur de $\mathrm{Cu}_{2} \mathrm{O}$ (sphère sombre) entouré d'une coquille de Zn0 (zone plus claire autour de la sphère). Synthèse réalisée dans l'azote liquide, entre deux électrodes de cuivre (1000 décharges), puis entre deux électrodes de zinc (1000 décharges).

Les décharges peuvent être réalisées dans des liquides diélectriques réactifs, comme l'eau qui permet de produire des oxydes (e.g. $2 \mathrm{Al}+3 \mathrm{H}_{2} \mathrm{O} \rightarrow \mathrm{Al}_{2} \mathrm{O}_{3}+3 \mathrm{H}_{2}$, lorsque les électrodes sont en aluminium), ou les alcanes qui donnent lieu, outre les nanoparticules issues de l'érosion des électrodes, à des nano-carbones (l'ensemble des formes nanométriques que le carbone peut adopter, comme par exemple des nanotubes, des fullerènes, du graphène ou autres), voire à du diamant si les décharges sont assez puissantes.

Elles peuvent aussi être réalisées dans des liquides non réactifs comme les liquides de gaz rares (argon ou hélium liquide) ou l'azote liquide. Ces liquides non réactifs offrent la possibilité de conserver la nature métallique des nanoparticules.

Grâce à la meilleure compréhension du comportement des décharges dans les liquides, la synthèse de nanoparticules peut être améliorée en :

- dissipant une charge plus faible par impulsion, de manière à réduire la pression du plasma sur le puits liquide et limiter la formation de particules submicrométriques ;

- exploitant la dissymétrie du courant qui oscille entre les électrodes pour chauffer de préférence une électrode plutôt que l'autre, et favoriser la formation d'alliages ; 
- favorisant les instabilités de bulles pour accroitre la surface d'échange gaz-liquide et permettre un transfert plus efficace des nanoparticules de la bulle vers le liquide. Ceci peut être réalisé, par exemple, en générant la décharge près d'une paroi, ce qui déclenche une impulsion de Kelvin (voir encadré) qui augmente l'interface gaz-liquide. En ce sens, l'utilisation de microréacteurs pourrait permettre de limiter l'agglomération des nanoparticules et tendre vers des distributions de taille plus proches de la monodispersité.

Il est alors possible de former (fig. 4) :

- des nanoparticules de métaux simples ou de leurs oxydes ;

- des nanocarbones, comme des nanofils, des nano-oignons (sortes de fullerènes imbriqués les uns dans les autres ayant l'aspect d'un oignon), des nanocornes (sortes de structures creuses de diamètre variable ayant la forme d'une corne), etc. ; - des structures dites cœur-coquille, où une nanoparticule d'un matériau donné est enveloppée dans une couche d'un matériau différent. Dans ce cas, on réalise une première série de décharges pour produire des particules du matériau des électrodes choisies ; puis on change d'électrodes et l'on réalise une nouvelle série de décharges pour produire dans le même liquide où se trouvent les premières nanoparticules en suspension une vapeur qui viendra les recouvrir pour former la coquille du composite élaboré. Cette vapeur peut également donner lieu à des nanoparticules simples, mais de manière d'autant plus marginale que la densité de nanoparticules formant le cœur de la structure est grande.

Si la formation de composites est assez simple, celle d'alliages métalliques est, en revanche, particulièrement complexe.

On peut, pour cela, choisir de travailler avec deux électrodes faites de matériaux différents. Dans ce cas, la première difficulté vient de ce que, pour des systèmes binaires comme, par exemple, le zinc et le cuivre qui constituent la base des laitons, les points de fusion des deux métaux sont très différents $\left(419^{\circ} \mathrm{C}\right.$ pour le premier contre $1085^{\circ} \mathrm{C}$ pour le second). Leurs fusions ne se produisent donc pas simultanément, ce qui limite leur interaction. Lorsque les points de fusion sont proches, les éléments ne sont pas nécessairement miscibles et l'on obtient au mieux des biparticules formées par la juxtaposition de deux particules de natures différentes. Enfin, lorsque les éléments sont miscibles, il se produit un phénomène de ségrégation de surface des éléments lorsque la taille des nanoparticules diminue suffisamment, ce qui modifie naturellement la composition des matériaux sélectionnés et surtout leur catalycité, c'est-à-dire leur propension à orienter certaines réactions chimiques qui peuvent se dérouler à leur surface.

Par ailleurs, il est possible d'utiliser directement des électrodes d'alliages métalliques. Dans ce cas, il est fréquent de former, outre la phase recherchée, des phases intermédiaires présentant différentes compositions, ce qui complexifie fortement la récupération de la phase attendue.

Dans tous les cas, les nanoparticules doivent être fonctionnalisées en surface après leur élaboration pour éviter leur coalescence. Cela permet de les conserver individuellement et donc de préserver les propriétés associées à leur taille nanométrique. Cette fonctionnalisation peut être réalisée, par exemple, en ajoutant des tensioactifs qui modifient la tension superficielle entre deux surfaces de nanoparticules. Cette ingénierie de surface est essentielle pour produire des nanofluides où les particules ne s'agglomèrent pas et donc ne décantent pas, mais restent en suspension. De tels fluides ont des propriétés fortement anisotropes sitôt que l'on applique un champ électrique, les nanoparticules s'alignant alors et modifiant par exemple la viscosité du milieu dans le sens du champ appliqué. Ce principe a été utilisé récemment pour équiper les chars de l'armée américaine de nouveaux systèmes hydrauliques permettant, au moyen d'une boucle d'asservissement contrôlant l'intensité du champ électrique appliqué, d'absorber parfaitement les chocs d'une route accidentée.

\section{Références}

1 A. Starikovskiy et al., "Non-equilibrium plasma in liquid water: dynamics of generation and quenching", Plasma Sources Sci. Technol., 20 (2011) 024003.

2• M. Pekker et al., "Initiation stage of nanosecond breakdown in liquid", J. Phys. D: Appl. Phys., 47 (2014) 025502.

3• L. Schaper et al., "Vapour layer formation by electrica discharges through electrically conducting liquidsmodelling and experiment", Plasma Sources Sci. Technol,, 20 (2011) 034003

\section{Conclusion}

La redécouverte des décharges dans des liquides diélectriques pour des applications inédites a permis d'ouvrir de nouvelles perspectives. Même si une description satisfaisante des premiers instants du claquage n'est toujours pas disponible, des progrès importants ont été accomplis.

Ainsi, la description de l'interface décharge-liquide a beaucoup bénéficié d'expériences réalisées en microgravité. Actuellement, de nombreux travaux s'orientent vers la formation de décharges dans des bulles présentes dans les liquides. On a en effet remarqué qu'il était possible de créer des décharges dans un gaz confiné dans un liquide et que cela pouvait être utilisé pour valoriser certains gaz, comme le méthane notamment. On a également observé qu'il était possible de synthétiser des molécules rares, comme les «diamantoïdes " qui constituent un petit groupe de composés tridimensionnels ayant une structure semblable au diamant et pouvant servir à la synthèse de nouvelles molécules thérapeutiques. Comme dans certaines conditions on observe une propagation de la décharge à l'interface gaz-liquide, des travaux ont été entrepris pour étudier la formation de décharges sur des bulles de liquides, et même sur des mousses. Ceci pourrait trouver des applications dans le domaine des tensio-actifs utilisés dans des industries comme les biotechnologies ou l'emballage, et dont l'efficacité pourrait être améliorée grâce à la synthèse d'espèces actives produites par les décharges [5].

Les perspectives offertes par les décharges dans les liquides sont considérables, ce qui motive de nombreuses études fondamentales en vue d'améliorer notre maîtrise de ces milieux complexes.

\footnotetext{
4 P. Wagener et al., "Dynamics of silver nanoparticle formation and agglomeration inside the cavitation bubble after pulsed laser ablation in liquid", Phys. Chem. Chem. Phys., 15 (2013) 3068-3074.

$5 \bullet Y u$. Akishev et al., "The interaction of positive streamers with bubbles floating on a liquid surface", Plasma Sources Sci. Technol., 24 (2015) 065021.
} 